\title{
Reflets
}

Revue ontaroise d'intervention sociale et communautaire

\section{Restructuration des soins de santé : impact sur les personnes âgées en Ontario}

\section{Manon Lemonde et Johanne Pomerleau}

Volume 2, numéro 2, automne 1996

Vieillir à l'aube de l'an 2000

URI : https://id.erudit.org/iderudit/026135ar

DOI : https://doi.org/10.7202/026135ar

Aller au sommaire du numéro

Éditeur(s)

Reflets : Revue ontaroise d'intervention sociale et communautaire

ISSN

1203-4576 (imprimé)

1712-8498 (numérique)

Découvrir la revue

Citer cet article

Lemonde, M. \& Pomerleau, J. (1996). Restructuration des soins de santé : impact sur les personnes âgées en Ontario. Reflets, 2(2), 128-135.

https://doi.org/10.7202/026135ar

Tous droits réservés (C) Reflets : Revue ontaroise d'intervention sociale et communautaire, 1996
Ce document est protégé par la loi sur le droit d'auteur. L'utilisation des services d'Érudit (y compris la reproduction) est assujettie à sa politique d'utilisation que vous pouvez consulter en ligne.

https://apropos.erudit.org/fr/usagers/politique-dutilisation/ 


\title{
Restructuration des soins de santé: impact sur les personnes âgées en Ontario
}

\author{
Manon Lemonde et Johanne Pomerleau \\ École des sciences infirmières, Université Laurentienne, Sudbury
}

\section{Introduction}

En Ontario, l'arrivée du gouvernement conservateur a entraîné d'importants remaniements dans tous les services publics. En effet, ce gouvernement tente par tous les moyens de redresser la situation économique de la province. Ces remaniements influencent d'une façon ou d'une autre tous les citoyens de l'Ontario. Notre réflexion portera tout particulièrement sur les conséquences engendrées par les remaniements au sein des services de santé destinés aux personnes âgées. Les opinions émises ainsi que les observations ont été recueillies dans le cadre d'un projet de recherche longitudinal, ayant pour but d'évaluer une intervention en promotion de la santé auprès des personnes âgées. Les résultats de ces rencontres ont permis de comprendre à quel point les personnes âgées seront tout particulièrement affectées par la restructuration des services de santé.

La campagne politique des conservateurs prônait le redressement budgétaire, afin que le gouvernement parvienne à équilibrer son budget global. Pour le parti conservateur, ce thème impliquait une réduction des coûts des services de santé fournis 
«...les personnes âgées ont plus de difficulté à s'adapter au changement d'attitude véhiculé par la restructuration...» aux résidents de l'Ontario. Cela devait être accompli par une stabilisation des coûts, voire une réduction liée à l'adoption d'un plan de restructuration des services de santé, en vue d'une meilleure répartition des ressources humaines. En principe, ce changement devait favoriser l'accessibilité aux services sur tout le territoire de l'Ontario et en augmenter l'efficacité. L'utilisation appropriée des ressources humaines et technologiques est donc nécessaire pour atteindre cet objectif.

L'impact de ces changements rapides va probablement affecter davantage les personnes âgées. En effet, l'abolition de la loi sur la création d'Organismes de services polyvalents, la modification de la loi sur le remboursement des médicaments, la restructuration et le virage ambulatoire affectent spécifiquement les personnes âgées. Dans le Nord, les conséquences seront d'autant plus grandes que l'accès aux services de santé est déjà réduit.

Malgré les bonnes intentions du gouvernement en matière de réduction du déficit, il faut être sensible à l'impact des compressions budgétaires sur les personnes âgées, car elles forment un groupe économique considéré comme étant très vulnérable. En effet, les personnes âgées ont plus de difficulté à s'adapter au changement d'attitude véhiculé par la restructuration, où l'on cherche à rendre chaque citoyen responsable de sa santé. Cette nouvelle orientation va à l'encontre du système dans lequel ces personnes ont grandi, c'est-à-dire à l'intérieur d'une société qui assumait la responsabilité de leur santé grâce à l'État providence. Aujourd'hui, le message est totalement différent. Les personnes âgées doivent prendre les moyens de demeurer autonomes le plus longtemps possible, sans nécessairement bénéficier de ressources financières et matérielles, et surtout sans avoir de formation susceptible de favoriser leur autonomie à mesure qu'elles vieillissent. Ainsi, il devient pratiquement impossible d'atteindre cet objectif. Afin de surmonter ce problème, les personnes âgées se doivent d'apprendre à travailler avec d'autres personnes et de former des partenariats qui leur permettront de garder ou maintenir leur niveau d'autonomie. 


\section{Redressement budgétaire}

"...est-ce-que les

personnes âgées vont se procurer les médicaments prescrits nécessaires pour se maintenir en santé?»
Les coupures budgétaires affectent tout particulièrement les personnes âgées qui ont un revenu fixe. Elles doivent suppléer aux restrictions économiques imposées par les gouvernements. Par exemple, avec la nouvelle réglementation sur l'assurancemédicament, les personnes âgées devront assumer les frais encourus pour faire remplir une ordonnance, y compris les frais professionnels du pharmacien. Ce changement peut représenter une dépense supplémentaire annuelle de 100,00 \$ par personne ${ }^{1}$. Cette nouvelle modalité entraînera des changements divers dans les habitudes de consommation de médicaments chez les personnes âgées. Les effets les plus plausibles sont le risque accru de surdosage, la surconsommation de médicaments en vente libre, l'accumulation de médicaments périmés, et l'échange de médicaments avec d'autres personnes. Ces comportements sont déjà observables chez les personnes âgées. À plus long terme, la question est la suivante: est-ce-que les personnes âgées vont se procurer les médicaments prescrits nécessaires pour se maintenir en santé? Il est possible de penser que l'impact de cette nouvelle réglementation des soins de santé entraînera la hausse des coûts du système et des conséquences graves pour la santé des personnes âgées, plutôt que la diminution des coûts. Il devient de plus en plus difficile de prôner l'autonomie et de responsabiliser les individus malades si on ne peut leur assurer un minimum de soutien.

Il est vrai qu'il faut éliminer la duplication des services de santé, tout en facilitant leur accès. Toutefois, ni les intervenants ni la population n'ont été préparés à réagir efficacement à tous ces changements rapides. Il n'est donc pas surprenant que les personnes âgées expriment, lors des rencontres associées au projet d'intervention en promotion de la santé, leurs craintes, leur insatisfaction, voire même leur résistance à l'égard des professionnels de la santé et des personnes impliquées dans des décisions d'ordre politique. Si aucune action n'est prise pour modifier 
la situation, ces comportements pourraient entraver toutes les démarches de restructuration et de prise en charge personnelle de la santé.

Pour être et demeurer en bonne santé, il faut être capable de s'adapter, d'identifier et d'utiliser les ressources appropriées pour répondre à ses propres besoins de santé. Aujourd'hui, cette capacité d'adaptation ne repose pas uniquement sur chaque individu. Il faut donner la possibilité de faire des choix éclairés, soit au terme d'un processus de réflexion où les intervenants du domaine indiquent les ressources disponibles, et où les individus pourront choisir le moyen qui leur convient pour demeurer en bonne santé.

\section{Soins de santé primaires}

"...les intervenants de la santé travaillent de concert avec les gens ... pour leur permettre d'atteindre ainsi un plus haut niveau d'autonomie."
La philosophie des soins de santé primaires, au moment de sa mise en œuvre dans les collectivités, préconise que les gens, par leur participation directe, identifient leurs besoins de santé, leurs problèmes de santé ou sociaux, ainsi que la ligne de conduite qui serait acceptable (World Health Organization, 1978). Ce niveau d'engagement signifie que les membres de la communauté seront impliqués dans la planification, l'organisation, la gestion et l'évaluation des soins. La conséquence directe d'une telle philosophie est d'augmenter la responsabilité de tous les membres de la communauté, sans pour autant les pénaliser pour les choix qu'ils feront et qui pourraient influencer leur niveau de santé. Selon cette approche, les intervenants de la santé travaillent de concert avec les gens, en s'appuyant sur la connaissance personnelle de leur santé pour leur permettre d'atteindre ainsi un plus haut niveau d'autonomie.

Les principes mis de l'avant dans les soins de santé primaires (Alma-Ata, 1978) sont les suivants: accessibilité aux soins de santé dispensés à des coûts abordables pour la société, soins adaptés aux besoins de santé des individus, groupes et communautés, 
"...l'approche préconisée par le gouvernement conservateur ne reçoit pas l'appui escompté...» adaptation et utilisation de la technologie de façon appropriée et soins conçus et gérés par les communautés. Ces principes ont quelque peu redéfinis dans la restructuration en cours. Il est donc important de les revoir, et surtout d'élaborer des moyens pour faciliter leur mise en œuvre à partir de ressources diminuées.

Les réactions observées auprès de la communauté avec laquelle nous travaillons vont dans le même sens que cette affirmation:

... les soins de santé primaires ne pourront prendre de sens et se développer si l'on ne prend en compte, et si l'on ne mesure pas l'ampleur du changement de conception philosophique, culturelle, sociale et économique qu'ils représentent, tant pour les personnels sanitaires de toutes catégories que pour la population (Collière, 1996: 265).

Actuellement, l'approche préconisée par le gouvernement conservateur ne reçoit pas l'appui escompté, puisque la santé est vue, par la population, comme un bien de consommation auquel elle a droit et qu'il faut préserver malgré les compressions budgétaires.

Les personnes âgées francophones se sentent menacées par les changements initiés par le gouvernement. Elles craignent pour les acquis obtenus après de longues batailles, notamment l'accessibilité à des services de santé en français. Ces derniers ne sont pas garantis dans les plans de restructuration. Cette situation aurait des conséquences graves pour les aînés unilingues francophones, en limitant davantage l'accès aux ressources, ou encore en éliminant les ressources existantes. D'ailleurs, les observations faites auprès des personnes âgées francophones de Sudbury révèlent qu'elles manifestent leur insatisfaction en refusant de participer à certains programmes d'intervention en promotion de la santé. Elles justifient leur geste sur la raison d'être de ces programmes, qui est de favoriser la prise en charge. Les personnes âgées considèrent que le gouvernement ne pourrait plus réduire les services aux personnes âgées si elles refusent de se prendre en main.

Malgré les bonnes intentions du gouvernement ou des fonctionnaires responsables de l'équilibre budgétaire du système de 
"...les principes

suivants sont énoncés dans la constitution: universalité, transférabilité, administration publique, accessibilité ainsi que gratuité des soins de santé et hospitaliers." santé, il ne faut pas oublier que la province de l'Ontario est tenue de respecter la loi fédérale concernant les soins de santé. En effet, les coupures ou les initiatives de restructuration correspondentelles aux principes de base de cette loi? Comme le soulignait Betkowski (1993), il faut réaliser que la solution ne réside pas seulement dans le fait de déplacer le poids des coûts générés par le système de soins de santé des gouvernements fédéral et provincial vers les individus, mais de repenser le système de santé. Il faut également se souvenir que les principes suivants sont énoncés dans la constitution: universalité, transférabilité, administration publique, accessibilité ainsi que gratuité des soins de santé et hospitaliers. Ce dernier principe est celui qui a été le plus modifié au cours des dernières années, avec l'adoption de règlements qui permettent la facturation de certains services comme les frais d'hébergement en soins prolongés, la diminution progressive ou le retrait de services qui étaient antérieurement gratuits, et le ticket modérateur. Tous ces efforts n'ont pas entraîné de stabilisation ou de baisse des coûts de la santé; au contraire la situation s'est aggravée, perturbant ainsi la qualité des services offerts. Il est temps de reconnaître que tous les citoyens sont responsables des coûts de la santé. Afin de spécifier davantage cette responsabilité, il est important de l'associer à la notion d'imputabilité qui n'a jamais été liée à l'élaboration et à la gestion de nos services de santé, ce qui a entrainé des abus par les professionnels de la santé et les citoyens dans l'utilisation des ressources humaines et technologiques.

\section{Discussion}

Après avoir décrit succinctement l'impact de la restructuration sur les personnes âgées, il faut identifier la ou les personnes capables de déterminer si les conséquences sont acceptables pour les personnes âgées et les professionnels de la santé. Les soins de santé canadiens et la philosophie des soins de santé primaires ont en commun un principe de base, celui de l'autodétermination. 
"...chaque individu a le droit de définir ce qui est bien et acceptable pour maintenir sa santé...»
«En ce qui concerne les personnes âgées, l'impact de la restructuration, ainsi que la position économique précaire de la province, auront un effet direct sur leur qualité de vie.»
En effet, chaque individu a le droit de définir ce qui est bien et acceptable pour maintenir sa santé, ainsi que ce qui est mauvais. Toutefois, la situation actuelle montre que dans la majorité des cas, ce ne sont pas les individus qui prennent les décisions concernant leur santé. La prise de décision et la notion de choix éclairé, qui fondamentalement sont le reflet du droit à l'autodétermination de chaque personne, ont été institutionnalisés par les professionnels de la santé et le système de soins. Alors, estil possible de croire que l'on donnera aux personnes âgées l'occasion de déterminer et d'exprimer ce dont elles ont besoin pour être en bonne santé? En fait, leur permettra-t-on de définir et exprimer clairement ce qui, pour elles et leur communauté, est bon ou mauvais pour la santé? Actuellement, le processus de restructuration semble se limiter à la compression des dépenses, en diminuant le nombre de lits d'hôpitaux et en fermant certains hôpitaux. Cette situation ramène toutes les discussions à la répartition des ressources humaines et technologiques, donc aux notions d'accès et d'équité, qui demeurent les principes directeurs de notre système de santé et des soins de santé primaires. Cependant, vu la rapidité avec laquelle ces changements se produisent, il n'est pas certain que le processus mis de l'avant jusqu'à maintenant permette aux individus d'avoir le temps de s'adapter et d'apprendre à vivre dans ce nouvel environnement. Actuellement, il n'est pas évident que la province ait adopté un plan stratégique qui favoriserait une phase de transition, tant pour les personnes âgées, la population en général que pour les professionnels de la santé.

En ce qui concerne les personnes âgées, l'impact de la restructuration, ainsi que la position économique précaire de la province, auront un effet direct sur leur qualité de vie. Les choix qu'elles auront à faire pourraient être limités en raison de leur situation économique et de la région qu'elles habitent.

L'enjeu de la restructuration et du virage ambulatoire est énorme, non seulement pour les personnes âgées, mais pour toute personne qui a besoin et aura besoin de soins dans la communauté. Sans que cela ne soit ouvertement discuté, les notions d'universalité, d'accès et de gratuité des services de santé sont 
redéfinies pour devenir de plus en plus étroites et de moins en moins équitables. Si cette tendance se maintient, il faudra que les gens soient consultés de façon à pouvoir participer à la redéfinition de ces notions, sans oublier le rôle et le niveau de responsabilité que chacun des acteurs doit assumer dans ce système de santé restructuré.

C'est pourquoi, il faut continuer à chercher de nouvelles façons de stimuler et d'éduquer la population, jeune et moins jeune, ainsi que les professionnels de la santé, de façon à créer des partenariats qui s'avéreront nécessaires pour surmonter les conditions difficiles qui s'annoncent.

Note

1. Selon les auteures, cette nouvelle réglementation serait l'équivalent d'un ticket modérateur. Le but d'un ticket modérateur est de diminuer l'abus de consommation en pénalisant le comportement abusif.

\section{Bibliographie}

BETKOWSKY, N.J. (1993) . «Healthy Communities and the Canada Health Act: Do they Work at Counter Purpose?» dans B. Rogers, (editor) Proceedings - Healthy Communities in Action You Make a Difference,. Sudbury, Ontario:The Sudbury Roundtable on Health, Economy and Environment, 19-24.

COLLIERE, M.F. (1996). Soigner.. le premier art de la vie, Paris, InterÉditions.

WORLD HEALTH ORGANIZATION (1978). Alma-Ata 1978: Primary Health Care. Health for All series, no. 1, Genève: World Health Organization. 